\title{
Implicações do Ambiente Físico de Aprendizagem na Formação de Mestres Profissionais em Administração
}

\author{
Implications of the Physical Learning Environment in the Formation of \\ Professional Masters in Administration \\ Implicaciones del entorno físico de aprendizaje en la formación de maestrías \\ profesionales en administración
}

MARY DAYANE SOUZA SILVA

Orcid Id: http://orcid.org/0000-0003-3144-7588

Universidade Federal do Pernambuco

\begin{abstract}
Resumo: O pensamento acadêmico contemporâneo sugere que o professor não pode limitar-se apenas a dar aulas. É necessário também que crie um ambiente de aprendizagem no qual o aluno seja levado a compartilhar conhecimento em sala de forma aberta, fluida e espontânea, acompanhado por igual crescimento de sua compreensão como elemento ativo-participativo no aprendizado construído a partir desse ambiente. A presente pesquisa objetivou compreender as implicações do ambiente físico de aprendizagem na formação de mestres profissionais de administração. Os dados foram coletados por meio de entrevistas e foram analisados qualitativamente. Os resultados indicam que a disposição do mobiliário de forma circular em sala de aula, articulada a partir da organização mobiliar formalmente estabelecida, constitui um ambiente eficazmente dinâmico de aprendizagem em que há um nítido desenvolvimento da interação de forma mais ativa e participativa dos alunos no encadeamento da condução da aula, oportunizando a gestão de espaços de ensino benéficos à aprendizagem.
\end{abstract}

Palavras-chave: Ambiente Físico de Aprendizagem; Ambiente de Aprendizagem; Formação de Mestres Profissionais.

\begin{abstract}
Contemporary academic thinking suggests that the teacher cannot be limited to teaching. It is also necessary to create a learning environment in which the student is led to share knowledge in the classroom in an open, fluid and spontaneous way, accompanied by an equal growth in his understanding as an active-participative element in the learning built from that environment. This research aimed to understand the implications of the physical learning environment in the training of professional masters of administration. The data were collected through interviews and were analyzed qualitatively. The results indicate that the arrangement of the furniture in a circular way in the classroom, articulated from the formally established furniture organization, constitutes an effectively dynamic learning environment in which there is a clear development of the interaction in a more active and participatory way of the students in the chain. conducting the class, enabling the management of teaching spaces beneficial to learning
\end{abstract}

Keywords: Physical Learning Environment; Learning Environment; Training of Professional Masters. 
Resumen: El pensamiento académico contemporáneo sugiere que el profesor no puede limitarse a enseñar. También es necesario crear un ambiente de aprendizaje en el que el alumno sea conducido a compartir conocimientos en el aula de manera abierta, fluida y espontánea, acompañado de un igual crecimiento en su comprensión como elemento activo-participativo en el aprendizaje construido desde ese ambiente. Esta investigación tuvo como objetivo comprender las implicaciones del entorno físico de aprendizaje en la formación de maestros profesionales en administración. Los datos se recolectaron mediante entrevistas y se analizaron cualitativamente. Los resultados indican que la disposición del mobiliario de forma circular en el aula, articulada a partir de la organización del mobiliario formalmente establecida, constituye un ambiente de aprendizaje efectivamente dinámico en el que bay un claro desarrollo de la interacción de forma más activa y participativa de los alumnos de la cadena. la conducción de la clase, posibilitando la gestión de espacios didácticos beneficiosos para el aprendizaje.

Palabras-clave: Entorno de aprendizaje físico; Ambiente de aprendizaje; Formación de Maestros Profesionales.

\section{INTRODUÇÃO}

Um ambiente centrado no desenvolvimento do aprendizado deve enfatizar os indivíduos como construtores do seu conhecimento, assim como a importância do contexto na formação destes e a natureza essencial da experiência nesse processo. Em espaços de ensino tradicionais, os alunos muitas vezes não têm a oportunidade de desenvolver uma aprendizagem ativa, pois apenas absorvem as informações que lhe são apresentadas passivamente (FENWICK, 2003), apesar de estarem inevitavelmente envolvidos em alguma forma de direcionamento de aprendizagem no ambiente.

Os indivíduos em idade adulta devem estar inseridos em um ambiente que desenvolva um contexto educacional capaz de melhorar o seu processo de aprendizagem e que responda aos desafios profissionais e, ao fazê-lo, construa e transforme a compreensão do seu conhecimento e que não apenas os leve a adquirir e acumular informações (LOYENS; GIJBELS, 2008). Esse processo vai além das características curriculares, uma vez que reflete sobre a natureza da aprendizagem, a estrutura do ambiente e o papel dos sujeitos no processo de formação e aprendizagem (HANNAFIN; LAND, 1997).

O ambiente da sala de aula deve refletir essas características não só por facilitar a adoção de abordagens de aprendizagem, mas também por proporcionar aos alunos o aprimoramento de seus conceitos de autoaprendizagem (DART et al.,1999). O indivíduo, ao se envolver no processo de aprendizagem, deve ter a consciência de que também está inserido em contextos que podem ser benéficos a si próprio, uma vez que envolvem estratégias de ensino-aprendizagem diversificadas para ampliar as interações individuais e estimular a iniciativa e a participação de forma mais ativa em sala. Ambientes de aprendizagem eficazes 
devem oferecer aos sujeitos oportunidades de alcançar seu potencial como aprendizes (WARGER; SERVE; DOBBIN, 2009; LOMAS; OBLINGER, 2006). Tal ambiente de aprendizagem difere em alguns pontos de um espaço gerido de forma tradicional, pois não foca principalmente na transferência de informação, mas na obtenção de experiências reais; o diálogo não é baseado em um monólogo, mas na interação entre professor e aluno; o foco é na orientação continuada; não considera só a cognição, mas também as emoções; e centra-se na criação de mais opções dinâmicas para o aprendizado e na promoção da participação mútua (KUIJPERS; MEIJERS; GUNDY, 2011).

A proposta de construir um ambiente de aprendizado mais dinâmico e participativo pelos alunos leva à reflexão sobre como sujeitos em idade adulta, enquanto aprendizes, veem a ruptura com o layout utilizado tradicionalmente em sala de aula (professor atuando como ponto central no processo de aprendizagem) e a formação de ambientes mais dinâmicos, nos quais a estrutura mobiliar da sala é disposta em círculos no intuito de estimular a interação e a participação ativa dos alunos durante seu processo de aprendizado, oportunizando a gestão de espaços de ensino benéficos. Esse esforço direcionado para a compreensão de como adultos respondem à gestão e às transformações do espaço físico que compõem o ambiente de aprendizagem leva ao seguinte questionamento de pesquisa: como o ambiente físico de aprendizagem contribui para a formação de mestres profissionais de administração? Para responder a esta questão, tem-se como objetivo compreender as implicações do ambiente físico de aprendizagem na formação de mestres profissionais de administração.

Nas seções que seguem, apresentam-se o ambiente de aprendizagem, sua dimensão física de aprendizagem, além dos procedimentos metodológicos adotados na pesquisa e as considerações inferidas sobre o fenômeno estudado.

\section{AMBIENTE DE APRENDIZAGEM}

Existe pouco consenso sobre o que constitui um ambiente de aprendizagem, que tipos de limites podem ou devem ser estabelecidos, quais são os elementos-chave e como eles interagem, o que pode ser resultado da complexidade envolvida nos processos de ensinar e aprender (DAY, 2009). O termo ambiente de aprendizagem não é novo, uma vez que engloba significados e definições nas mais diversas situações e contextos. Abrange recursos de aprendizagem e tecnologias, meios de ensino, modos de aprendizagem, e conexões com contextos sociais e globais, incluindo ainda dimensões comportamentais e culturais humanas, além do papel fundamental da emoção no aprendizado (WARGER; SERVE; DOBBIN, 
2009). Logo, sua compreensão como conceito envolve pesquisas que variam da perspectiva metodológica educacional, psicológica, para a mais sociológica ou antropológica (RADOVAN; MAKOVEC, 2015).

O termo contexto de aprendizagem ou ambientes de aprendizagem, até duas décadas atrás, não aparecia na literatura educacional dominante (RADOVAN; MAKOVEC, 2015). O termo tem sido usado livremente no discurso educacional por causa do uso emergente das tecnologias da informação para fins educativos (KUUSKORPI; FINLAND; GONZÁLEZ, 2011), bem como do reconhecimento do ambiente como um local onde a aprendizagem acontece, por meio da percepção comum dos sujeitos sobre ele (FRASER, 2002), ao se referir a todos os aspectos do contexto que influenciam o cumprimento de metas de aprendizagem dentro de um conteúdo específico ou área temática (KOCK; SLEEGERS; VOETEN, 2004).

O campo de desenvolvimento do ambiente de aprendizagem tem sido moldado por várias figuras de relevante influência nos últimos anos. Diversos estudos foram realizados para examinar a influência que o ambiente teria, por exemplo, sobre a fixação de metas (MAEHR; MIDGLEY, 1991), estratégias de ensino (VARUGHESE; FEHRING, 2009), características de aprendizagem em grupos, classes e ambientes escolares (PERRY; VAN de KAMP, 2000; SHERA, 2014) que contribuíram com o incremento de pesquisas e estudos sobre a temática (WOLF; FRASER, 2008). Essa diversificação de estudos durante os últimos anos sobre o ambiente de aprendizagem levou o campo a sofrer um notável crescimento, que possibilitou pôr à disposição uma variedade de questionários válidos e aplicáveis amplamente no intuito de realizar avaliações das percepções dos alunos de seu ambiente de aprendizagem em sala de aula (WOLF; FRASER, 2008). O ambiente passou a ser, de alguma forma, determinado pela percepção dos indivíduos que agem sobre ele, o que estaria intimamente relacionada com o seu resultado de aprendizagem (RADOVAN; MAKOVEC, 2015; WOLF; FRASER, 2008).

Ressalta-se ainda que o ambiente de aprendizagem está situado dentro de um contexto específico de ensino-formação que, por sua vez, está inserido num contexto ainda mais amplo, por envolver fundamentos sociais, psicológicos e tecnológicos na aprendizagem, fatores que se deve ter em mente tanto na compreensão do ambiente quanto ao se considerar uma atividade de aprendizagem (TISDELL, 1995). Assim como a aprendizagem, o ambiente não é um conceito unidimensional, uma vez que existem múltiplas dimensões que devem ser entendidas holisticamente, a fim de captar a verdadeira natureza da relação entre essas duas dimensões (HIEMSTRA, 1991), bem como suas influências sobre os 
indivíduos. Para fins desta pesquisa, optou-se em estudar a dimensão física do ambiente. A seção a seguir traz algumas considerações acerca do ambiente físico de aprendizagem.

\section{AMBIENTE FÍSICO DE APRENDIZAGEM}

O contexto do ensino tem ampliado seu alcance no intuito de não depender única e exclusivamente de um modelo que tem por base um aprendizado estático e mecanizado, uma vez que, no âmbito educacional, especialmente, a aprendizagem ganhou configurações que transformaram a forma como os indivíduos aprendem, já que estes podem influenciar-se mutuamente e sofrer influências (MERRIAM; BIEREMA, 2014). Toda aprendizagem ocorre em um ambiente com características físicas quantificáveis e perceptíveis, ou seja, a todo momento os sujeitos são envolvidos pelas informações do meio, que podem afetá-los emocionalmente, com consequências cognitivas e comportamentais importantes (GRAETZ, 2006).

Ao abordar a contribuição da estrutura física no aprendizado dos indivíduos, delimitam-se alguns pontos de relevância significativa como: mobiliário, disposição das cadeiras em sala, acústica, tal como um ambiente que de alguma maneira exerça influência na aprendizagem (KUUSKORPI; FINLAND; GONZÁLEZ, 2011; LOMAS; OBLINGER, 2006). A composição de um ambiente de aprendizagem tem por objetivo potencializar a forma como o aprendizado dos indivíduos se desenvolve. Sua disposição física deve proporcionar um ambiente agradável, transformador, experiencial, construtivo, adequadamente propício para a ocorrência dos diversos tipos de aprendizados formais, não-formais e informais. Tais características têm o potencial de fomentar o desenvolvimento do aprendizado na dimensão física do ambiente de aprendizagem.

Em um sentido mais restrito, um ambiente físico de aprendizagem é visto como uma sala de aula convencional, que normalmente têm requisitos relativamente simples como uma linha de visão, uma boa acústica e um ponto focal na parte da frente da sala (LOMAS; OBLINGER, 2006). Em um sentido mais amplo, é entendido como uma combinação de sistemas formais e informais de educação, por meio dos quais a aprendizagem tem lugar dentro e fora do contexto de aprendizagem (KUUSKORPI; FINLAND; GONZÁLEZ, 2011).

Em um ambiente de aprendizagem, a estrutura física faz referência aos espaços, equipamentos e ferramentas de dentro da instituição, que precisam ser equipados com estações de trabalho e áreas modulares com assentos confortáveis, versáteis, resistentes, duráveis e fáceis de reparar. Deve ser possível adaptar o 
mobiliário a diferentes configurações e métodos de trabalho que contribuam para apoiar a aprendizagem individual e em grupo (KUUSKORPI; FINLAND; GONZÁLEZ, 2011).

O conceito de ambiente físico de aprendizagem tem evoluído para uma estrutura ainda mais complexa, ao incluir atividade de didática mais dinâmica e estratégias de ensino ativas, além de estimular uma maior reflexividade por parte dos alunos, onde os sujeitos podem participar no processo de aprendizagem, tanto direta como indiretamente por meio da reflexão posterior das atividades estabelecidas em sala (KUUSKORPI; FINLAND; GONZÁLEZ, 2011). A dimensão física refere-se ao espaço real por meio do qual a aprendizagem acontece (MERRIAM; BROCKETTT, 2007). Assim, quando os ambientes físicos oferecem recursos e possibilidades que apoiam novos métodos de ensino e objetivos de aprendizagem, tanto professores quanto alunos passam a vivenciar um espaço dinâmico, no qual os métodos de trabalho são orientados ao contexto e integração do grupo. A seção a seguir apresenta o caminho metodológico percorrido para a realização da pesquisa.

\section{PROCEDIMENTO METODOLÓGICO}

Esta seção do trabalho possui como proposta apresentar os procedimentos metodológicos utilizados para a estruturação do referencial teórico, coleta de dados e análises dos resultados.

A presente pesquisa caracteriza-se como uma investigação de cunho qualitativo. A escolha pela pesquisa qualitativa justifica-se por não se utilizar de um conjunto de técnicas específicas, mas sim de um enfoque que busca compreender fenômenos sociais (VERGARA, 2005), uma vez que se almeja entender como as pessoas constroem, atribuem significados e interpretam as suas experiências (MERRIAM, 2009; GODOI; BALSINI, 2006). O interesse está em compreender os fenômenos sociais, tendo como foco os significados construídos pelos sujeitos a partir das experiências vividas ou sentidas nos diversos contextos em que se inserem.

Participaram dessa pesquisa ex-alunos do Mestrado Profissional em Administração (MPA) da Universidade Federal da Paraíba, num total de onze entrevistados das turmas 1, 2 e 3. A escolha por ex-alunos de turmas diferentes teve o intuito de trazer uma visão ampla das experiências vividas nesse ambiente em períodos distintos do MPA.

As entrevistas foram realizadas a partir de um roteiro semiestruturado elaborado a partir do referencial proposto, e tiveram duração aproximada de 40 min cada. Salienta-se que os nomes dos entrevistados não foram identificados por 
questões de privacidade e respaldo profissional. Assim, nos resultados da pesquisa, estes são identificados como E1, E2, E3, E4, E5, E6, E7, E8, E9, E10, E11. A seleção dos entrevistados se deu de forma intencional e por acessibilidade, tendo como critério de escolha os ex-alunos que concluíram o curso até 2014, por serem as turmas iniciais a concluírem o programa e se tornarem mestres profissionais.

Para a apreciação dos resultados, utilizou-se a análise compreensivainterpretativa proposta por Silva (2006), a qual ocorre a partir de cinco momentos: no primeiro, realizou-se a transcrição da entrevista, leitura e releitura do protocolo da entrevista e a transformação destes em protocolo codificado. No segundo momento, foi feita a releitura dos protocolos codificados para identificação de temas. No terceiro, realizou-se o agrupamento dos relatos dos sujeitos entrevistados em categorias de análise. No quarto momento, a estruturação dos resultados em um texto acerca de cada tema das categorias de análise. E, por fim, no quinto momento, ocorreu a análise compreensiva-interpretativa dos resultados.

$\mathrm{Na}$ seção seguinte são apresentados os resultados da pesquisa, bem como a análise compreensiva-interpretativa realizada a partir dos discursos.

\section{RESULTADOS DA PESQUISA}

O ambiente é de alguma forma determinado pelas percepções dos alunos que atuam nesse meio, as quais se relacionam diretamente com os resultados da aprendizagem (RADOVAN; MAKOVEC, 2015; WOLF; FRASER, 2008), podendo facilitar tal aprendizagem, ao proporcionar um ambiente agradável e colaborativo para que o aprendizado seja construído de forma ativa pelos indivíduos, ou podem levar a um contexto que cause certa inconstância ou ansiedade, ao lidar com algo novo ou fora do comumente esperado do ponto de vista individual.

Um ambiente que viabilize o diálogo, que seja agradável em termos ergonômicos, um local com boa iluminação, arejado, amplo, confortável, com recursos e equipamentos tecnológicos, em que haja compartilhamento de conhecimento, que transmita confiança e facilite a ocorrência do aprendizado, além da figura do professor como parte integrante do ambiente, são alguns dos elementos ressaltados pelos entrevistados em seus discursos a respeito do que consideram como um ambiente físico propício para a aprendizagem.

Outra questão relacionada à composição física do ambiente refere-se à disposição das cadeiras em sala. Para Chism (2006), a visão da sala de aula com cadeiras dispostas de forma enfileirada com uma mesa na frente e, por trás dela, um quadro negro, transmite uma abordagem pedagógica segundo a qual o instrutor ou professor fala ou demonstra e o aluno escuta e observa. Isso fica 
evidente nos relatos a seguir: "As cadeiras em frente ao professor não é um ambiente muito propício para haver um diálogo, porque acaba ficando um ao lado do outro" (E7). "[...] mas o espaço físico que nos foi ofertado foi esse: uma sala de aula comum com cadeiras dispostas, até mesmo as cadeiras eram dispostas da maneira tradicional, em que o professor ficava na frente e os alunos sentados" (E3). "O espaço físico de aprendizagem é simples, mas é adequado, em minha opinião. Ou seja, não restringe o aprendizado. Tem aparelho de ar-condicionado, quadro branco, projetor etc., e as cadeiras eram enfileiradas como em uma sala de aula normal" (E5).

A disposição das cadeiras de maneira tradicional, enfileiradas, permite a criação de uma relação unilateral no ambiente. Uma forma de potencializar a dinâmica da sala e do aprendizado nesse contexto que faria mais sentido, segundo Chism (2006), seria construir um ambiente suscetível a reconfigurações rápidas que suportem diferentes tipos de atividades em sala. Essa composição estrutural do ambiente físico de aprendizagem foca em atuar como palco para a construção do conhecimento, possível por meio da visão de que a composição física não se limita somente a características ergonômicas como luminosidade, temperatura do ambiente, entre outras, mas também inclui o layout e a disposição circular dos móveis no contexto da sala de aula.

A formação das cadeiras em forma de círculos contribui na dinâmica da sala, além da influência no comportamento e na participação dos alunos na aula. Esse tipo de disposição modular tira a centralidade da figura do professor, permitindo uma visão do grupo de forma igual como um todo e uma maior interatividade face a face entre os indivíduos em sala de aula. Essa percepção fica evidente nos relatos dos entrevistados a seguir: "Em círculos com outras pessoas lado a lado você pode olhar olho no olho e interagir mais" (E10). "Houve algumas aulas que houve essa modificação nessa centralidade que foi tirada do professor e foram abertos círculos onde houve uma maior interação" (E5). "Houve em algumas aulas essa mudança dessa logística da sala, mas em outras não, era um ambiente tradicional de professor e aluno" (E11).

Essa visão tem ganhado cada vez mais foco nas discussões acadêmicas, devido ao reconhecimento da necessidade da criação de ambientes de aprendizagem que permitam uma interação mais direta entre professor e alunos (CHISM, 2006). A formação em círculos no ambiente da aula possibilita maior troca de ideias, proximidade entre os indivíduos tanto internamente ao contexto da sala de aula como externamente a ele, por meio da motivação e participação dos alunos de forma dinâmica e enriquecedora.

As interações por meio da disposição circular têm grande potencial de favorecer a aprendizagem a partir da discussão, da troca de ideias, dos relatos 
de experiências e do compartilhamento do conhecimento de forma individual e coletiva. A circularidade na disposição das cadeiras no contexto do ensino como sendo favorável à aprendizagem pode ser percebida nas falas que seguem: "Na sala de aula geralmente fazíamos círculos, trocávamos ideias e tinha até mesmo a questão da acústica porque muitas vezes não entendíamos o que fulano estava querendo dizer e tal, muitas vezes se acabava fazendo comentários equivocados porque você entendeu uma coisa diferente daquilo que ele disse realmente" (E2). "A gente utilizou algumas vezes que eu me recordo a formação em U e em círculos também para formar grupos e duplas e essa interação em círculos eram muito boas e favoreciam a aprendizagem, em que as cadeiras elas não ficavam dispostas só na forma como elas estavam por ser uma sala de aula, já que não era um auditório com cadeiras fixas e tal” (E4). "Às vezes tinham (sic) professores que gostavam de fazer círculos. Como eu gostava de debates, a formação em círculo era bem mais favorável e fazia com que todos se motivassem e participassem mais e se sentissem mais aptos a falar porque estavam em círculos, aí a aula ficava mais enriquecedora com a contribuição de cada pessoa" (E1).

Esse tipo de formação circular no contexto da sala de aula apresenta várias oportunidades que possibilitam usos diversificados do ambiente físico, a partir das relações estabelecidas por meio da reconfiguração do espaço no desenvolvimento da aprendizagem, as quais podem direcionar a projeção de espaços mais dinâmicos e interativos, ao permitir o acesso e a aplicação de diferentes abordagens em sala. As relações estabelecidas no ambiente físico permitem maior interação e a construção de um ambiente mais enriquecedor (RADOVAN; MAKOVEC, 2015).

A importância que a disposição do mobiliário exerce sobre o aprendizado reflete-se sobre a motivação e o interesse participativo em sala estimulado pela formação de círculos, que remetem a uma visão do todo de forma igual, enriquecendo ainda mais a dinâmica contextual da sala e, dessa forma, proporcionando uma maior integração entre as partes constituintes do ambiente. Essa percepção fica evidente na fala a seguir: "Quando você coloca as cadeiras dispostas dessa outra maneira, ou seja, em círculo, você possibilita essa interação até mesmo no olhar, você se sente mais próximo" (E9).

Para Chism (2006), a disposição mobiliar do ambiente físico de aprendizagem deve ser considerada com o mesmo foco na aprendizagem que a formação curricular na formação dos indivíduos. O ambiente físico deve ser percebido como um fator crítico, que afeta o aprendizado e exerce sobre este um forte impacto, à medida que pode criar um ambiente mais dinâmico, propício para o desenvolvimento do diálogo e mais interativo entre os alunos-professor-alunos. Além disso, tem o potencial de fomentar uma maior integração dos indivíduos 
com o contexto em que se almeja desenvolver a aprendizagem e com a realidade das pessoas na compreensão do todo.

Tal visão é perceptível no relato do entrevistado a seguir: "Tinha professor que chegava e fazia uma roda com todos os alunos e tentava discutir a partir das experiências dos alunos [...], mas eu acredito que a aprendizagem era mais verdadeira por causa daquele diálogo e da realidade das pessoas e daquela interação entre elas e com o professor" (E8).

Construir no ambiente de aprendizagem um espaço, a partir da reconfiguração do mobiliário em sala de aula, possibilita uma visão do todo de maneira uniforme, ampla, ao permitir que os alunos se vejam mais envolvidos, mais livres e mais impulsionados a se expressarem e a exporem suas opiniões. A disposição do mobiliário no formato circular em sala de aula propicia a criação de um sentimento de inserção maior no contexto de aprendizagem, levando o aluno a adotar uma postura mais ativa no seu próprio processo.

Assim, o aprendizado proporcionado na dimensão física do ambiente encaminha os indivíduos para a vivência de ações voltadas ao convívio coletivo, de forma relacional, em que o conhecimento é compartilhado a partir dos relacionamentos e das relações interpessoais construídas em sala de aula. Além disso, as experiências de aprendizagem a partir da interação oportunizada pela formação circular do mobiliário em sala de aula têm grande potencial transformador do conhecimento dos sujeitos ao originar reflexões acerca de suas próprias ações, tendo por base a visão de outros ao relatarem suas experiências de vida durante suas atuações profissionais ou até mesmo no campo pessoal. A Figura 01 a seguir retrata bem a descrição do ambiente físico de aprendizagem a partir da formação de círculos em sala. 
Figura 01 - Visão do Ambiente de aprendizagem dos alunos em sala de aula a partir da formação de círculos

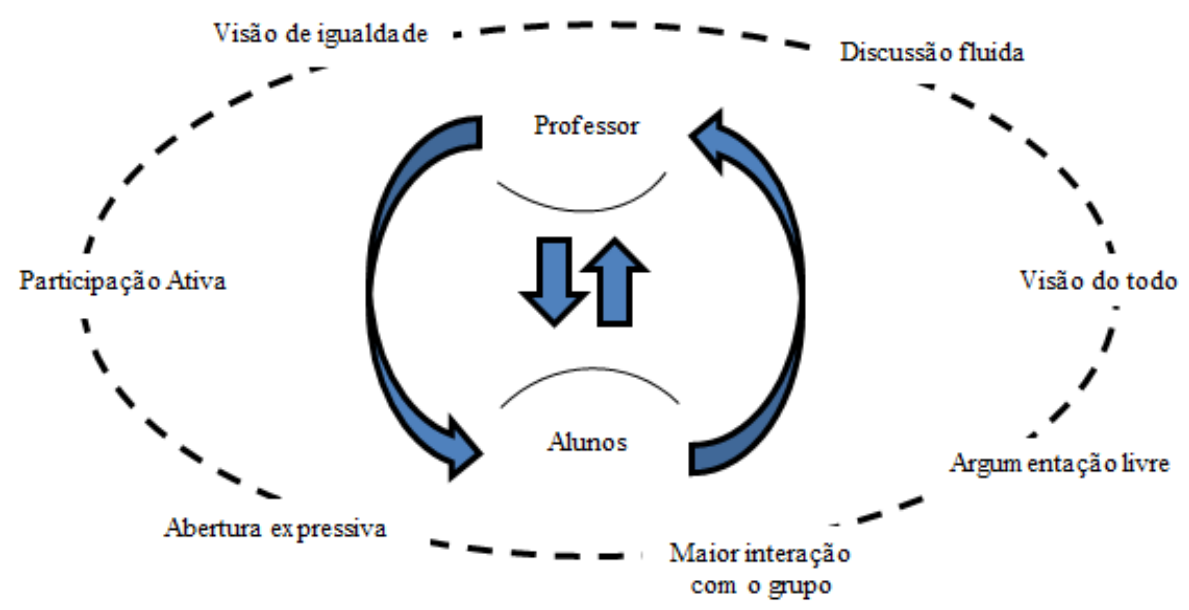

Fonte: Elaboração própria, 2019.

A formação de círculos no ambiente de aprendizagem permitiu tanto ao professor quanto aos alunos estabelecerem uma relação mais interativa, ao romper com a estrutura hierarquizada imposta pela disposição tradicional do mobiliário, permitindo criar um ambiente no qual o compartilhamento das experiências e do conhecimento são oportunizadas pela amplitude da nova relação. Além disso, viabiliza aos alunos um ambiente de aprendizagem marcado pela igualdade, que origina a visão do todo e no qual as discussões podem fluir espontaneamente. Tal configuração tende a provocar maior interação com o grupo, dando abertura expressiva e possibilidade de argumentar livremente e, consequentemente, fomentando uma participação mais ativa em seu processo de ensino-aprendizagem dentro deste novo ambiente. Essa transformação somente foi possível a partir da aproximação da relação professor-aluno nesse espaço.

\section{CONSIDERAÇÕES FINAIS}

Os resultados desta investigação revelam que a reconfiguração da arquitetura mobiliar física do ambiente contribui para uma aprendizagem vivencial balizada por aspectos estruturais com potencial transformacional na forma como o conhecimento é construído a partir das condições experienciadas nesse espaço físico. 
A criação de um ambiente em que alunos e professores sejam capazes de se envolver verdadeiramente em relações que ajudem a ambos a se sentirem bem e emocionalmente à vontade em sala para a realização de suas atividades tem se configurado cada vez mais como um ponto crucial para o processo de ensino-aprendizagem dos indivíduos. O ambiente a ser ofertado aos indivíduos deve viabilizar o surgimento de reações emocionais positivas, no qual os alunos sintam-se motivados, seguros e confortáveis durante o processo de aprendizagem, sendo, assim, um contexto fértil para o desenvolvimento do conhecimento. Tal percepção permite uma visão reflexiva de que a forma como se molda o ambiente físico transforma o aprendizado a ser desenvolvido e, consequentemente, a forma como a aprendizagem acontece nesse ambiente.

A partir da reflexão acerca do ambiente físico no aprendizado, foi possível caracterizá-lo como sendo um ambiente no qual o papel do docente assume destaque no processo de formação. A figura do professor é vista como parte responsável na construção do conhecimento, como um dos protagonistas principais durante a formação dos indivíduos, assim como nas experiências vivenciadas no decurso do processo de formação no programa.

Ademais, a tentativa de proporcionar um ambiente favorável ao processo de aprendizagem é tarefa complexa. Tal iniciativa exige que educadores de profissionais adultos não atentem somente para os espaços físicos nos quais a aprendizagem tem lugar, mas também para um conjunto de atitudes, percepções, conhecimentos, valores, crenças, entendimentos e normas que são compartilhadas culturalmente e exercem influências sobre comportamentos, ações, decisões e a forma como indivíduos conduzem sua atuação social e profissional.

\section{REFERENNCIAS}

CHISM, N. V. N. Challenging traditional assumptions and rethinking learning spaces. In: OBLINGER, D. G. (Org.). Learning spaces. Educause, 2006.

DART, B. et al. Classroom learning environments and students' approaches to learning. Learning Environments Research, v.2, pp. 137-156, 1999.

DAY, K. Creating and sustaining effective learning environments. All Ireland Journal of Teaching and Learning in Higher Education - AISHE, v.1, n.1, 2009.

FENWICK, T. J. Learning through experience: troubling orthodoxies and intersecting questions. Florida: Krieger Publishing Company, 2003. 
FRASER, B. J. Learning environments research: yesterday, today and tomorrow. In: GOH, S. C.; KHINE, M. S. (Eds.). Studies in educational learning environments: an international perspective. World Scientific Publishing, 2002.

GODOI, C. K.; BALSINI, C. P. V. A pesquisa qualitativa nos estudos organizacionais brasileiros: uma análise bibliométrica. In: GODOI C. K.; BANDEIRA-DE-MELLO, R.; SILVA, A. B. (Orgs.). Pesquisa qualitativa em estudos organizacionais: paradigmas, estratégias e métodos. São Paulo: Saraiva, 2006.

HANNAFIN, M. J.; LAND, S. M. The foundations and assumptions of technology-enhanced student-centered learning environments. Instructional Science, v. 25, pp. 167-202, 1997.

KOCK, A.; SLEEGERS, P.; VOETEN, M.J. M. New learning and the classification of learning environments in secondary education. Review of Educational Research, v. 74, n.2, pp. 141-170, 2004.

KUUSKORPI, M.; FINLAND, K.; GONZÁLEZ, N. C. The future of the physical learning environment: school facilities that support the user. CELE Exchange, 2011.

LOMAS, C.; OBLINGER, D. G. Student practices and their impact on learning spaces. In: OBLINGER, D. G (Org). Learning spaces. Educause, 2006.

LOYENS, S. M. M; GIJBELS, D. Understanding the effects of constructivist learning environments: introducing a multi-directional approach. Instructional Science, v. 36, pp. 351-357, 2008.

MAEHR, M. L.; MIDGLEY, C. Enhancing student motivation: a schoolwide approach. Educational Psychologist, v. 26, n.3, pp. 399-427, 1991.

MERRIAM, S. B. Qualitative research: a guide to design and interpretation. San Francisco: Jossey-Bass, 2009.

MERRIAM, S. B; BIEREMA, L. L. Adult learning: linking theory and practice. São Francisco: Jossey-Bass, 2014. 
PERRY, N. E; VAN de KAMP, K. J. Creating classroom contexts that support young children's development of self-regulated learning. International Journal of Educational Research, v. 33, n. 8, pp. 821-842, 2000.

RADOVAN, M.; MAKOVEC, D. Adult learners' learning environment perceptions and satisfaction in formal education. Case study or four east-european countries. International Education Studies, v. 8, n. 2, 2015.

TISDELL, E. J. Creating inclusive adult learning environments: insights from multicultural education and feminist pedagogy. Columbus, Ohio: ERIC - Clearinghouse on Adult, Career, and Vocational Education, 1995.

VARUGHESE, V. K.; FEHRING, H. Effects of students' approaches to learning on performance in two pedagogical environments. International Education Studies, v. 2, n. 4, pp. 10-14, 2009.

WARGER, T.; SERVE, E.; DOBBIN, G. Learning environments: where space, technology, and culture converge. Educause Learning Initiative, 2009.

WOLF, S. J.; FRASER, B. J. Learning environment, attitudes and achievement among middle-school science students using inquiry-based laboratory activities. Research in Science Education, v. 38, pp.321-341, 2008.

\section{Mary Dayane Souza Silva}

Doutora em Admininistração na Universidade Federal do Pernambuco (UFPE). E-mail: m.dayane.silva@gmail.com 